\title{
Normal modes of magnetic flux tubes and dissipation
}

\author{
K. Karami ${ }^{1}$, S. Nasiri ${ }^{1,2}$, and Y. Sobouti ${ }^{1,3}$ \\ ${ }^{1}$ Institute for Advanced Studies in Basic Sciences, Gava Zang, Zanjan 45195, Iran \\ 2 Department of Physics, Zanjan University, Zanjan, Iran \\ 3 Center for Theoretical Physics and Mathematics, AEOI, PO Box 11345-8486, Tehran, Iran \\ e-mail: Nasiri@iasbs.ac.ir; Sobouti@iasbs.ac.ir
}

Received 19 April 2002 / Accepted 25 June 2002

\begin{abstract}
Wave propagation in a zero- $\beta$ magnetic flux tube with a discontinuous Alfvèn speed at its surface is considered. The problem is reduced to solving a wave equation for the projection of the magnetic perturbation along the axis of the cylinder. The mathematical formalism is identical with that for the propagation of electromagnetic waves in optical fibers with a varying index of refraction in the cross section of the fiber. The dispersion relation is solved in its full generality and three wave numbers are assigned to the normal modes of the cylinder. There is a lower cutoff for the longitudinal wave number along the cylinder axis and an upper cutoff for the radial wave number. Eigenfrequencies and eigenfields (i.e. the magnetic and velocity fields of modes) are calculated. Resistive and viscous dissipation rates have mathematically identical forms, differing only in their being inversely proportional to the Lundquist and Reynolds numbers, respectively. These rates as well as the energy densities are obtained for each mode and are commented on.
\end{abstract}

Key words. Sun: corona - magnetohydrodynamics (MHD) - Sun: magnetic fields - Sun: oscillations

\section{Introduction}

Since the discovery of the hot solar corona about sixty years ago, different theories of coronal heating have been put forward and debated. Recent observations, however, have indicated the existence of magnetohydrodynamic (mhd) waves and their damping in coronal loops and suggest they may be sources of heat supply to the corona. In their analysis of observations by the Transition Region and Coronal Explorer (TRACE), Nakariakov et al. (1999) reported the detection of spatial oscillations in five coronal loops with periods ranging from 258 to $320 \mathrm{~s}$. They interpreted them as global mhd standing waves driven by solar flares in the adjacent active regions. TRACE also detected decaying oscillations in a long, $(130 \pm 6) \times 10^{6} \mathrm{~m}$, and thin, $(2 \pm 0.36) \times 10^{6} \mathrm{~m}$, bright coronal loop in the $171 \AA$ emission lines of FeIX. The decay time was $(14.5 \pm 2.7) \mathrm{min}$ for an oscillation of $(3.9 \pm 0.13)$ millihertz. All these observations indicate strong dissipation of the wave energy that may be the cause of coronal heating.

Resonant absorption of Alfvèn waves in coronal inhomogeneities was first suggested by Ionson (1978) as a nonthermal mechanism of heating. He pointed out the importance of the density and magnetic field gradients in dissipating the wave energy. Ionson's conclusions received further support from the more extensive calculations of Hollweg (1984). Wentzel (1979a,b) was among the early investigators studying the propagation and dissipation of mhd waves along surfaces of

Send offprint requests to: K. Karami, e-mail: Karami@iasbs.ac .ir discontinuity of Alfvèn speeds. Wilson (1979, 1980) studied the vibrational modes of flux sheaths and flux tubes embedded in compressible but unstratified atmospheres and obtained a general dispersion relation. Roberts (1981a,b) proposed the occurrence of magnetoacoustic surface waves along magnetic interfaces. Edwin \& Roberts (1983) elaborated on the dispersion relation for a magnetic cylinder embedded in a magnetic environment typical of that of the solar photosphere and corona. Roberts et al. (1984) found two distinct time scales (long acoustic and short Alfvèn ones) for the propagation of magnetoacoustic waves in coronal inhomogeneities. Steinolfson et al. (1986) were concerned with the role of viscous and resistive dissipations on surface waves in cases of both continuous and discontinuous variations of Alfvèn speeds. Davila (1987) and Steinolfson \& Davila (1993) did much analytic and numerical work on resonant absorption and of their resistive dissipation. Ofman et al. $(1994,1995)$ included viscous dissipation in their analysis and concluded that the shear viscous dissipation is of the same magnitude as the resistive heating. Contribution of the compressional viscosity, however, was found to be insignificant.

Here we study a cylindrical magnetic flux tube with a discontinuous Alfvèn speed across the surface of cylinder. The model is essentially the same as that of Edwin \& Roberts (1983) but without limiting it to slender tubes. In Sect. 2 we reduce the problem to solving a wave equation for the component of the magnetic field along the cylinder axis and discuss the relevant boundary conditions. In Sect. 3 we elaborate 
on the dispersion relation, give its graphical and numerical solutions, and introduce cutoffs. In Sect. 4 we discuss resistive and viscous dissipations. In Sects. 5 and 6 we give further numerical and graphical results. Section 7 is devoted to concluding remarks. Lengthy formulae are collected in Appendix A. Purely transverse modes, with no longitudinal components in magnetic and velocity fields, have certain peculiarities. They are touched upon in Appendix B.

\section{Formulation of the problem}

More often than not, magnetohydrodynamic (mhd) waves propagating in coronal loops have, mathematically, the same structure as the light signals propagating in optical fibers and/or dielectric resonators, a point addressed by Nakariakov $\&$ Roberts (1995) and Nakariakov (2000). For example, in a cylindrical fiber lying in the $z$-direction with a graded or steplike index of refraction, the electromagnetic fields $\boldsymbol{E}$ and $\boldsymbol{B}$ can be expressed in terms of $E_{z}$ or $B_{z}$ depending on whether the mode in question is a transverse magnetic or a transverse electric one. Similarly, in a low- $\beta$ magnetic flux tube with graded or step-like Alfvèn speed, propagation of fast magnetoacoustic waves can be reduced to solving a wave equation for the $z$-component of the perturbation in the magnetic field. In both cases the differential equations for $z$-components of fields are identical. The boundary conditions, however, differ. See e.g. Ghatak \& Thyagarajan (1998) for light propagation in optical fibers.

\subsection{Equations of motion}

The linearized mhd equations for a zero- $\beta$ plasma are

$$
\frac{\partial \delta \boldsymbol{v}}{\partial t}=\frac{1}{4 \pi \rho}\{(\nabla \times \delta \boldsymbol{B}) \times \boldsymbol{B}+(\nabla \times \boldsymbol{B}) \times \delta \boldsymbol{B}\}+\frac{\eta}{\rho} \nabla^{2} \delta \boldsymbol{v},
$$

$\frac{\partial \delta \boldsymbol{B}}{\partial t}=\nabla \times(\delta \boldsymbol{v} \times \boldsymbol{B})+\frac{c^{2}}{4 \pi \sigma} \nabla^{2} \delta \boldsymbol{B}$,

where $\delta \boldsymbol{v}$ and $\delta \boldsymbol{B}$ are the Eulerian perturbations in the velocity and magnetic fields; $\rho, \sigma, \eta$ and $c$ are the mass density, the electrical conductivity, the viscosity and the speed of light, respectively. Under coronal conditions the gas pressure is neglected in comparison with magnetic pressure (zero- $\beta$ approximation) and gravitational effects are omitted on account of the density scale height being much larger than dimensions of the flux tube. The viscosity is introduced in its non-tensorial form for simplicity. We will further assume that the dissipative terms in Eqs. (1) and (2) are much smaller. We will first solve the problem without these terms and re-introduce them later as small corrections in calculating contributions of the different modes to heating of the corona.

We let the flux tube lie along the $z$-axis and let $\rho=\rho\left(\boldsymbol{r}_{\perp}\right)$ and $\boldsymbol{B}=B\left(\boldsymbol{r}_{\perp}\right) \hat{\boldsymbol{z}}$, where $\boldsymbol{r}_{\perp}$ is the coordinate vector transverse to the magnetic field. That $\rho$ is independent of $z$ follows from the assumption of an unstratified tube. That $\boldsymbol{B}$ is a consequence of $\nabla . \boldsymbol{B}=0$. We further assume an exponential $z$ - and $t$ dependence, $\mathrm{e}^{i\left(k_{z} z-\omega t\right)}$, for any of the components $\delta \boldsymbol{v}$ and $\delta \boldsymbol{B}$. We take the following steps: $i$ ) Take the time derivative of Eq. (2) and substitute for $\partial \delta \boldsymbol{v} / \partial t$ from Eq. (1). ii) Decompose the resulting equation into its $z$ - and transverse components. We arrive at

$$
\begin{gathered}
{\left[\nabla_{\perp}^{2}-\left(k_{z}^{2}-\omega^{2} / v_{\mathrm{A}}^{2}\right)\right]\left(B \delta B_{z}\right)-\nabla_{\perp} \ln \left[B\left(k_{z}^{2}-\omega^{2} / v_{\mathrm{A}}^{2}\right)\right]} \\
\cdot \nabla_{\perp}\left(B \delta B_{z}\right)=0, \\
B \delta \boldsymbol{B}_{\perp}=-\frac{i k_{z}}{\left(k_{z}^{2}-\omega^{2} / v_{\mathrm{A}}^{2}\right)} \nabla_{\perp}\left(B \delta B_{z}\right),
\end{gathered}
$$

where $v_{\mathrm{A}}\left(\boldsymbol{r}_{\perp}\right)=\left[B^{2} / 4 \pi \rho\right]^{1 / 2}$ is the local Alfvèn speed. The viscous and resistive terms are neglected at this stage. Similarly decomposing Eq. (1) into its $z$ - and transverse components and eliminating $\delta B_{\perp}$ appearing in them by Eq. (4) gives

$$
\begin{aligned}
& \rho \delta v_{z}=\frac{1}{4 \pi} \frac{k_{z} v_{\mathrm{A}}^{2}}{\omega\left(k_{z}^{2} v_{\mathrm{A}}^{2}-\omega^{2}\right)} \nabla_{\perp} \ln B \cdot \nabla_{\perp}\left(B \delta B_{z}\right), \\
& \rho \delta \boldsymbol{v}_{\perp}=\frac{i}{4 \pi} \frac{\omega}{\left(k_{z}^{2} v_{\mathrm{A}}^{2}-\omega^{2}\right)} \nabla_{\perp}\left(B \delta B_{z}\right) .
\end{aligned}
$$

That all field components are expressible in terms of $\delta B_{z}$ holds for any geometry of the cross section of the flux tube and any dependence of $\boldsymbol{B}$ and $\rho$ on $\boldsymbol{r}_{\perp}$.

Equations (3)-(6) become singular if $k_{z}^{2} v_{\mathrm{A}}^{2}(r)-\omega^{2}=0$ at some point $r$. The singularity can, however, be removed by the introduction of any dissipation mechanism. This issue is addressed in ample detail by Ionson (1978), Davila (1987), Steinolfson \& Davila (1993), Ofman et al. (1994, 1995), Kivelson \& Russell (1997), Roberts \& Ulmschneider (1997), and Nakariakov (2000).

Hereafter, we consider a circular cylinder and use cylindrical coordinates $(r, \phi, z)$. For simplicity, the radius of the cylinder is taken as the unit of length. The length of the cylinder is assumed to be $\pi L$ in the same unit. To ensure periodicity in $z$ direction one must then have $k_{z}=l / L, l=1,2, \ldots$ We further assume a constant magnetic field throughout the space and a step-like mass density, $\rho_{\mathrm{i}}$ for $r<1$ and $\rho_{\mathrm{e}}<\rho_{\mathrm{i}}$ for $r>1$. The condition $\rho_{\mathrm{e}}<\rho_{\mathrm{i}}$ implies $v_{\mathrm{A}_{\mathrm{e}}}>v_{\mathrm{A}_{\mathrm{i}}}$ and it is necessary to have standing waves in the flux tube. Otherwise any perturbation in the fields will propagate away to infinity. With these simplifications, Eq. (3) reduces to

$$
\left(\frac{\mathrm{d}^{2}}{\mathrm{~d} r^{2}}+\frac{1}{r} \frac{\mathrm{d}}{\mathrm{d} r}+k^{2}-\frac{m^{2}}{r^{2}}\right) \delta B_{z}(r)=0, \quad k^{2}=\omega^{2} / v_{\mathrm{A}}^{2}-k_{z}^{2},
$$

where we have introduced a $\phi$-dependence $\mathrm{e}^{i m \phi}, m=0,1,2, \ldots$ Equation (7) is Bessel's equation. Inside the tube it could have solutions

$\delta B_{z}=J_{m}\left(k_{\mathrm{i}} r\right), \quad k_{\mathrm{i}}^{2}=\omega^{2} / v_{\mathrm{A}_{\mathrm{i}}}^{2}-k_{z}^{2}>0, \quad r<1$.

To have evanescent waves in the exterior region, $r>1$, the solution should be

$\delta B_{z}=A K_{m}\left(k_{\mathrm{e}} r\right), \quad k_{\mathrm{e}}^{2}=k_{z}^{2}-\omega^{2} / v_{\mathrm{A}_{\mathrm{e}}}^{2}>0, \quad r>1$,

where $K_{m}$ is a modified Bessel function with vanishing asymptotic behavior, $\mathrm{e}^{-i k_{\mathrm{e}} r}$, as $r \rightarrow \infty$. The matching constant $A$ is as yet unspecified. 


\subsection{Boundary conditions}

i) To avoid shock waves at $r=1$, the Lagrangian changes in pressure should be continuous. Here, on account of the zero- $\beta$ approximation and constancy of $B$ throughout space this reduces to the continuity of $\delta B_{z}$. Thus

$J_{m}\left(k_{\mathrm{i}}\right)=A K_{m}\left(k_{\mathrm{e}}\right)$.

ii) On account of $\nabla \cdot \delta \boldsymbol{B}=0, \delta B_{r}$ should be continuous at $r=1$. This, by Eqs. (4), (8) and (9) gives

$\left.\frac{1}{k_{\mathrm{i}}^{2}} \frac{\mathrm{d} J_{m}\left(k_{\mathrm{i}} r\right)}{\mathrm{d} r}\right|_{r=1}=-\left.\frac{A}{k_{\mathrm{e}}^{2}} \frac{\mathrm{d} K_{m}\left(k_{\mathrm{e}} r\right)}{\mathrm{d} r}\right|_{r=1}$

or $\frac{1}{k_{\mathrm{i}}} \frac{\mathrm{d} J_{m}\left(k_{\mathrm{i}}\right)}{\mathrm{d} k_{\mathrm{i}}}=-\frac{A}{k_{\mathrm{e}}} \frac{\mathrm{d} K_{m}\left(k_{\mathrm{e}}\right)}{\mathrm{d} k_{\mathrm{e}}}$.

A note on the behavior of $\delta v$ and $\delta \rho$ on the boundary of the cylinder is in order. From Eqs. (4) and (6) one finds $\delta v_{\perp}=$ $-\left(\omega / k_{z}\right)\left(\delta \boldsymbol{B}_{\perp} / B\right)$. Thus, $\omega, k_{z}$, and $B$ being constants, the continuity of $\delta B_{r}$ across the cylinder implies the continuity of $\delta v_{r}$. This is the boundary condition used by Edwin \& Roberts (1983). On the other hand the continuity equation written in the form $\delta \rho=-(i / \omega) \nabla .(\rho \delta \boldsymbol{v})$, gives $\delta \rho_{\mathrm{e}}-\delta \rho_{\mathrm{i}}=-(i / \omega)\left(\rho_{\mathrm{e}}-\rho_{\mathrm{i}}\right) \delta v_{r}$ at $r=1$. Therefore, $\delta \rho$ will vary discontinuously across the cylinder.

\section{Dispersion relation, classification of modes and cutoffs}

To simplify the notation further, we let $x=k_{\mathrm{i}}$ and $y=k_{\mathrm{e}}$. By Eqs. (8) and (9), $x$ and $y$ are related as follows

$y^{2}=C_{l}^{2}-\frac{\rho_{\mathrm{e}}}{\rho_{\mathrm{i}}} x^{2}$,

where

$C_{l}^{2}=\left(1-\rho_{\mathrm{e}} / \rho_{\mathrm{i}}\right)(l / L)^{2}, \quad l=1,2, \cdots$,

$\pi L$ in units of radius,

is a measure of the contrast between interior and exterior of the flux tube. Division of Eq. (11) by (10) now gives

$\frac{1}{x} \frac{J_{m}^{\prime}(x)}{J_{m}(x)}=-\frac{1}{y} \frac{K_{m}^{\prime}(y)}{K_{m}(y)}$,

where "'" on $J_{m}$ and $K_{m}$ indicates a derivative with respect to their appropriate arguments. Equation (14) is the dispersion relation. As an equation for $x$, it can be solved numerically and graphically. Once this is done, Eq. (10) can be used to find the matching constant $A$.

In thin flux tubes, $(L \gg 1)$, approximating $J_{m}(x)$ and $K_{\mathrm{m}}(y)$ as $(x / 2)^{m}$ and $(y / 2)^{-m}, m \geq 1$, gives $x=y$. This in turn leads to an approximate dispersion relation $\left(\omega / k_{z}\right)^{2}=v_{\mathrm{A}, \mathrm{av}}^{2}$ where the average Alfvèn speed, the same as the kink speed of Edwin \& Roberts (1983), is obtained from $v_{\mathrm{A}, \mathrm{av}}^{-2}=(1 / 2)\left(v_{\mathrm{A}_{\mathrm{i}}}^{-2}+v_{\mathrm{A}_{\mathrm{e}}}^{-2}\right)$. For $m=0$, however, this approach breaks down, because of the logarithmic behavior of $K_{0}(y)$ at small $y$. Thin flux tubes are studied in detail by Ionson (1978), Wentzel (1979a,b), Wilson (1979, 1980), Edwin \& Roberts (1983), Roberts et al. (1984), Hasan \& Sobouti (1987), Nasiri (1992), and Nakariakov et al. (1999).

\subsection{A graphical analysis of dispersion relation}

Here we study Eq. (14) in its generality and discuss the dependence of $\omega$ s on three wave numbers $n, m$ and $l$ pertaining to the $r, \phi$ and $z$-directions, respectively. In Fig. 1, the multi-branch solid curves with vertical asymptotes are the plots of the left hand side of Eq. (14). They branch out to infinity at zeros of $J_{m}(x)$, and intersect the $x$-axis at zeros of $J_{m}^{\prime}(x)$. Only the positive branches are needed and are shown in the figure. The dotted curves are the plots of the right hand side of Eq. (14). They are finite at $x=0$ and go to infinity at the asymptote $y^{2}=C_{l}^{2}-\left(\rho_{\mathrm{e}} / \rho_{\mathrm{i}}\right) x_{\max }^{2}=0$ or $x_{\max }=C_{l}\left(\rho_{\mathrm{i}} / \rho_{\mathrm{e}}\right)^{1 / 2}$. Beyond this asymptote, $y$ becomes imaginary and therefore unphysical. The intersections of the multi-branch curves with dotted ones are the solutions of Eq. (14). They are denoted by $x_{n m l}$, where $m=0,1,2, \ldots$, is the order of the Bessel function, as well as the azimuthal wave number associated with the $\phi$-coordinate; $n=1,2, \ldots, n_{\max }$, the roots of Eq. (14) and is the wave number along the $r$-coordinate. Finally $l=1,2,3, \ldots$, is the wave number in $z$-direction and enters through the contrast parameter $C_{l}^{2}$ of Eq. (13).

Asymptotic behavior of $x_{n m l}$ : let $\gamma_{m n}$ and $\gamma_{m n}^{\prime}$ be the $n$th root of $J_{m}(x)$ and $J_{m}^{\prime}(x)$, respectively. Asymptotically for higher roots one has $\gamma_{m n} \approx(2 n+m-1 / 2) \pi / 2$ and $\gamma_{m n}^{\prime} \approx(2 n+m-$ $3 / 2) \pi / 2$. From Fig. 1 for $m \geq 1$, it is clear that $\gamma_{m, n-1}<x_{n m l}<$ $\gamma_{m n}^{\prime}$. Using the asymptotic form of $\gamma$ s one finds

$x_{n m l}=\frac{1}{2}\left(\gamma_{m, n-1}+\gamma_{m n}^{\prime}\right)+\alpha_{l} \pi / 2 \approx\left(2 n+m-2+\alpha_{l}\right) \pi / 2$,

where $-1 / 2<\alpha_{l}<1 / 2$ is to be determined numerically. For $m=0, n$ in Eq. (15) should be replaced by $n+1$.

\subsection{Eigenfrequencies and eigenfunctions}

The mode frequencies in terms of $x_{n m l}$ can be written from the definition of Eq. (8):

$$
\begin{aligned}
\omega_{n m l}^{2}= & \omega_{\mathrm{A}}^{2}\left[x_{n m l}^{2}+(l / L)^{2}\right], \quad l=1,2, \cdots, \quad m=0,1, \cdots, \\
& n=1,2, \cdots, n_{\max }, \\
\approx & \omega_{\mathrm{A}}^{2}\left[\left(2 n+m-2+\alpha_{l}\right)^{2} \pi^{2} / 4+(l / L)^{2}\right], \quad m \geq 1,
\end{aligned}
$$

where $\omega_{\mathrm{A}}=v_{\mathrm{A}_{\mathrm{i}}}$ is the interior Alfvèn frequency. For $m=0$, $n$ in Eq. (16) should be replaced by $n+1$. The corresponding mode function for $\delta B_{z}$ is

$$
\begin{aligned}
\delta B_{z, n m l} & =J_{m}\left(x_{n m l} r\right) \mathrm{e}^{i\left(l z / L+m \phi-\omega_{n m l} t\right)}, \quad r<1, \\
& =A_{n m l} K_{m}\left(y_{n m l} r\right) \mathrm{e}^{i\left(l z / L+m \phi-\omega_{n m l} t\right)}, \quad r>1,
\end{aligned}
$$

where $y_{n m l}^{2}=C_{l}^{2}-\left(\rho_{\mathrm{e}} / \rho_{\mathrm{i}}\right) x_{n m l}^{2}$, and $A_{n m l}=J_{m}\left(x_{n m l}\right) / K_{m}\left(y_{n m l}\right)$; see Eqs. (8)-(10). The transverse $r$ - and $\phi$-components of $\delta \boldsymbol{B}$ and of $\delta \boldsymbol{v}$ are given in Appendix A, Eqs. (A.1)-(A.4), $\delta v_{z}=0$ in the present model.

A mode given by Eqs. (16), (17) and (A.1)-(A.4) is characterized by a trio of wave numbers $(n, m, l)$ that actually count the number of nodes or antinodes along $r, \phi$, and $z$ directions, respectively. This trio provides a suitable basis for the classification of modes. What in the literature are termed as sausage, kink and fluting modes, in the present analysis correspond to modes with $m=0,1$ and 2 or greater, respectively. 

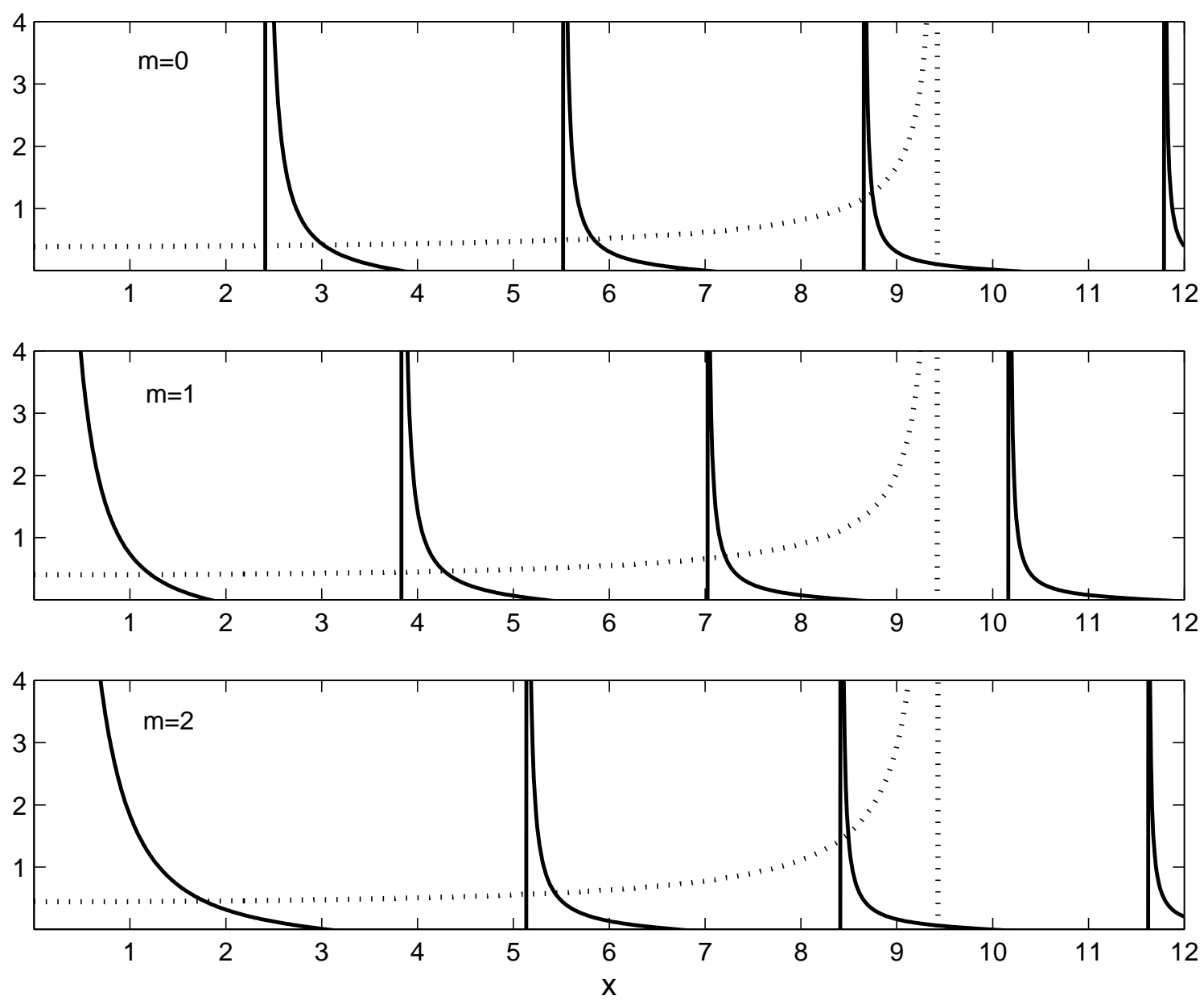

Fig. 1. The plots of the two sides of Eq. (14), the dispersion relation, as functions of $x$ for $m=0$ (sausage modes), $m=1$ (kink modes), and $m=2$ (fluting modes) with $l=100, C_{100}^{2}=8.9$, radius $=10^{3} \mathrm{~km}$, length $=10^{5} \mathrm{~km}$, and $\rho_{\mathrm{e}} / \rho_{\mathrm{i}}=0.1$. Left-hand side of dispersion relation, solid curve; right-hand side of the same equation, dotted curve. Intersections of solid and dotted curves, $x_{n m l}$, are tabulated in Tables 1-3.

\subsection{Cutoff wave numbers}

It was pointed out above that, for $m \geq 1, x_{n m l}$, whenever they exist, fall in the interval $\gamma_{m, n-1}<x_{n m l}<\gamma_{m n}^{\prime}$. By definition, $y_{n m l}^{2}=C_{l}^{2}-\left(\rho_{\mathrm{e}} / \rho_{\mathrm{i}}\right) x_{n m l}^{2}>C_{l}^{2}-\left(\rho_{\mathrm{e}} / \rho_{\mathrm{i}}\right) \gamma_{m, n-1}^{2} \geq 0$. This gives the lower cutoff, $l_{\min }$, for the longitudinal wave number

$l>l_{\min } \approx L \gamma_{m, n-1} /\left(\rho_{\mathrm{i}} / \rho_{\mathrm{e}}-1\right)^{1 / 2}, \pi L$ in units of radius.

For $m=0, \gamma_{0 n}$ should replace $\gamma_{0, n-1}$. The existence of a lower longitudinal cutoff, but only for $m=0$, was pointed out by Edwin \& Roberts (1983) and Roberts et al. (1984).

For a given $l>l_{\min }$ there exists an upper cutoff, $n_{\max }$, for the radial wave number. This can be obtained by examining $\gamma_{m n^{\prime}} \mathrm{S}$ and finding out which one is closest but smaller than $x_{\max }$; see again Fig. 1. Then

$$
\begin{aligned}
n_{\max } & =n^{\prime} & & \text { if } \quad m=0, \\
& =n^{\prime}+1 & & \text { if } \quad m \geq 1 .
\end{aligned}
$$

For large values of $x_{\max }(\approx 10)$, one may give an alternative expression,

$n_{\max } \approx \operatorname{integer}\left(x_{\max } / \pi\right)=\operatorname{integer}\left[\left(\rho_{\mathrm{i}} / \rho_{\mathrm{e}}-1\right)^{1 / 2} l / \pi L\right]$,

for, asymptotically the zeros of Bessel functions are spaced by $\pi$.

\section{Dissipative processes}

The finite conductivity and viscosity of plasma causes an exponential time decay of disturbances. For weak dissipations one may assume

$\delta \boldsymbol{B}^{\text {dissipative }}=\delta \boldsymbol{B}(\boldsymbol{r}) \mathrm{e}^{-(i \omega+\alpha) t}$,
$\delta \boldsymbol{v}^{\text {dissipative }}=\delta \boldsymbol{v}(\boldsymbol{r}) \mathrm{e}^{-(i \omega+\alpha) t}$,

where $\omega, \delta \boldsymbol{B}$, and $\delta \boldsymbol{v}$ on the right hand side are the solutions of Eqs. (16), (17) and (A.1)-(A.4) in the absence of dissipations. Substituting Eq. (21) in Eqs. (1) and (2), cancelling out the non dissipative terms, and keeping only the first order terms in $\alpha$, $c^{2} / 4 \pi \sigma$ and $\eta$ gives

$$
\begin{aligned}
-2 \alpha \delta \boldsymbol{B}= & \left(c^{2} / 4 \pi \sigma\right) \nabla^{2} \delta \boldsymbol{B} \\
& +(\eta / \rho)\left[\nabla^{2} \delta \boldsymbol{B}_{\perp}+\left(i \hat{z} / k_{z}\right) \nabla \cdot\left(\nabla^{2} \delta \boldsymbol{B}_{\perp}\right)\right] .
\end{aligned}
$$

Rewriting Eq. (22) for either the transverse or the $z$-component of a mode $(\mathrm{nml})$ and substituting for all quantities in terms of $\delta B_{z}$ gives

$$
\alpha_{n m l}=\left(\omega_{\mathrm{A}} / 4 \pi\right)\left(S^{-1}+R^{-1}\right)\left[x_{n m l}^{2}+(l / L)^{2}\right], \quad r<1,
$$

where the Lundquist number, $S=\left(\frac{4 \pi \sigma}{c^{2}}\right) /\left(\frac{2 \pi}{v_{\mathrm{A}_{\mathrm{i}}}}\right)$, is the ratio of the resistive time scale to the Alfvèn crossing time and the 
Reynolds number, $R=\left(\frac{\rho_{\mathrm{i}}}{\eta}\right) /\left(\frac{2 \pi}{v_{\mathrm{A}_{\mathrm{i}}}}\right)$ is the ratio of the viscous time scale to the Alfvèn crossing time. Equation (23) for $\alpha$ is appropriate for the inner region of the tube. A similar expression can be obtained for the outer region. It will be shown in Figs. 2 to 4 , however, that in the outer region all components of $\delta \boldsymbol{B}$ and $\delta \boldsymbol{v}$ are small and highly evanescent. They carry no significant amount of energy and can be ignored for all practical purposes.

The current density generated by $\delta \boldsymbol{B}$ is $\delta \boldsymbol{j}=(c / 4 \pi) \nabla \times \delta \boldsymbol{B}$. For a damped field of Eq. (21), this gives an ohmic heating rate of $\mathrm{d} \delta Q_{\text {ohmic }} / \mathrm{d} t=\frac{1}{2 \sigma} \int \mathrm{e}^{-2 \alpha t}|\delta \boldsymbol{j}(\boldsymbol{r})|^{2} \mathrm{~d}^{3} x$. Similarly the viscous heating rate is $\mathrm{d} \delta Q_{\text {visc }} / \mathrm{d} t=\frac{\eta}{2} \int \mathrm{e}^{-2 \alpha t}\left|\delta \boldsymbol{v} . \nabla^{2} \delta \boldsymbol{v}\right| \mathrm{d}^{3} x$. For a mode $(n m l)$ the integrands are given in Appendix A, Eq. (A.5). Both turn out to have identical mathematical form, leaving the ohmic and the viscous contribution to be proportional to $S^{-1}$ and $R^{-1}$, respectively. Correspondingly, the total dissipation time scale becomes $\tau_{\text {tot }}^{-1}=\left(\tau_{\text {ohmic }}^{-1}+\tau_{\text {visc }}^{-1}\right) \propto \alpha$.

The total heat $\delta Q$, generated mainly over one or two total dissipative time scales, is obtained by a further time integration of $\mathrm{d}\left(\delta Q_{\text {ohmic }}+\delta Q_{\text {visc }}\right) / \mathrm{d} t$. This, not surprisingly, turns out to be equal to the total energy initially vested in the wave in the form of kinetic and magnetic energies. Thus,

$$
\begin{aligned}
\delta Q & =\int\left(\frac{1}{2 \sigma}|\delta \boldsymbol{j}(\boldsymbol{r})|^{2}+\frac{\eta}{2}\left|\delta \boldsymbol{v} \cdot \nabla^{2} \delta \boldsymbol{v}\right|\right) \mathrm{d}^{3} x \int_{0}^{\infty} \mathrm{e}^{-2 \alpha t} \mathrm{~d} t, \\
& =\frac{1}{2} \int\left(\frac{1}{2} \rho|\delta \boldsymbol{v}|^{2}+\frac{1}{8 \pi}|\delta \boldsymbol{B}|^{2}\right) \mathrm{d}^{3} x, \\
& =\frac{\pi L}{8}\left(\frac{1}{x_{n m l}^{2}}+\frac{1}{y_{n m l}^{2}}\right) \\
& \times\left\{y_{n m l}^{2} J_{m}^{\prime 2}\left(x_{n m l}\right)+2(l / L)^{2} \frac{J_{m}\left(x_{n m l}\right) J_{m}^{\prime}\left(x_{n m l}\right)}{x_{n m l}}\right. \\
& \left.+\left[(l / L)^{2}-m^{2}-\left(\frac{1}{x_{n m l}^{2}}-\frac{1}{y_{n m l}^{2}}\right)(m l / L)^{2}\right] J_{m}^{2}\left(x_{n m l}\right)\right\} .
\end{aligned}
$$

The expression for the energy density of the wave is given in Eq. (A.6). If the total dissipative time scale, $\alpha^{-1}$, is short and the time evolution of heat generation is not of interest, one may take a short cut to the total generated heat by calculating the initial energy of the mode. Finally, the exact equality of the dissipated energy to the total initial energy of the wave should serve as a test of the consistency of the assumptions made throughout the present analysis and the correctness of the lengthy mathematical manipulations.

\section{Numerical results}

As typical parameters for a coronal loop, we assume radius $=$ $10^{3} \mathrm{~km}$, length $=10^{5} \mathrm{~km}, \rho_{\mathrm{e}} / \rho_{\mathrm{i}}=0.1, \rho_{\mathrm{i}}=2 \times 10^{-14} \mathrm{gr} \mathrm{cm}^{-3}$, $B=100 \mathrm{G}, \sigma=225 \times 10^{6} \mathrm{~s}^{-1}$, and $\eta / \rho=1.6 \times 10^{6} \mathrm{~m}^{2} \mathrm{~s}^{-1}$. For such a loop one finds $v_{\mathrm{A}_{\mathrm{i}}}=2000 \mathrm{~km} \mathrm{~s}^{-1}, v_{\mathrm{A}_{\mathrm{e}}}=6400 \mathrm{~km} \mathrm{~s}^{-1}$, $\omega_{\mathrm{A}}=2 \mathrm{rad} \mathrm{s}^{-1}, C_{l}^{2}=89 \times 10^{-5} l^{2}, S=R=2 \times 10^{4}$. The roots $x_{n m l}$ and $y_{n m l}$ are calculated and displayed in Tables 1, 2 and 3 for $m=0,1$, and 2 , respectively. In each table three radial mode numbers $n=1,2$ and 3, and several longitudinal wave numbers, $l$, are considered. Note the positions of cutoffs in Tables 1-3. For example, in Table 1, for $n=1,2,3$,
Table 1. Values of $x_{n m l}$ and $y_{n m l}$ for $m=0$ (sausage) modes with radius $=10^{3} \mathrm{~km}$, length $=10^{5} \mathrm{~km}, \rho_{\mathrm{i}}=2 \times 10^{-14} \mathrm{gr} \mathrm{cm}^{-3}$ and $\rho_{\mathrm{e}} / \rho_{\mathrm{i}}=$ 0.1 . These values give $C_{l}^{2}=89 \times 10^{-5} l^{2}$. No mode can exist in the dashed region of the table.

\begin{tabular}{ccccccc}
\hline \hline$l$ & \multicolumn{3}{c}{$x_{n m l}$} & & \multicolumn{4}{c}{$y_{n m l}$} \\
\hline 25 & - & - & - & - & - & - \\
26 & 2.4191 & - & - & 0.1282 & - & - \\
58 & 2.7718 & - & - & 1.4919 & - & - \\
59 & 2.7793 & 5.5314 & - & 1.5250 & 0.1962 & - \\
91 & 2.9757 & 5.7883 & - & 2.5465 & 2.0049 & - \\
92 & 2.9807 & 5.7937 & 8.6588 & 2.5777 & 2.0436 & 0.1883 \\
100 & 3.0190 & 5.8349 & 8.7444 & 2.8264 & 2.3442 & 1.1196 \\
\hline & $n=1$ & $n=2$ & $n=3$ & $n=1$ & $n=2$ & $n=3$ \\
\hline
\end{tabular}

Table 2. Same as Table 1, for $m=1$ (kink) modes.

\begin{tabular}{ccccccc}
\hline \hline$l$ & \multicolumn{3}{c}{$x_{n m l}$} & & \multicolumn{3}{c}{$y_{n m l}$} \\
\hline 1 & 0.0284 & - & - & 0.0284 & - & - \\
40 & 0.7865 & - & - & 1.1671 & - & - \\
41 & 0.7984 & 3.8369 & - & 1.1968 & 0.1545 & - \\
74 & 1.0827 & 4.1281 & - & 2.1809 & 1.7803 & - \\
75 & 1.0890 & 4.1347 & 7.0237 & 2.2108 & 1.8157 & 0.2701 \\
100 & 1.2183 & 4.2783 & 7.2106 & 2.9583 & 2.6589 & 1.9237 \\
\hline & $n=1$ & $n=2$ & $n=3$ & $n=1$ & $n=2$ & $n=3$ \\
\hline
\end{tabular}

Table 3. Same as Table 1, for $m=2$ (fluting) modes.

\begin{tabular}{ccccccc}
\hline \hline$l$ & \multicolumn{3}{c}{$x_{n m l}$} & & \multicolumn{3}{c}{$y_{n m l}$} \\
\hline 1 & 0.0284 & - & - & 0.0284 & - & - \\
54 & 1.2383 & - & - & 1.5627 & - & - \\
55 & 1.2541 & 5.1400 & - & 1.5922 & 0.2243 & - \\
89 & 1.6768 & 5.3727 & - & 2.6016 & 2.0404 & - \\
90 & 1.6865 & 5.3785 & 8.4230 & 2.6315 & 2.0775 & 0.3380 \\
100 & 1.7769 & 5.4337 & 8.4934 & 2.9299 & 2.4378 & 1.2985 \\
\hline & $n=1$ & $n=2$ & $n=3$ & $n=1$ & $n=2$ & $n=3$ \\
\hline
\end{tabular}

one finds $l_{\min }=25,58,91$, respectively. Conversely for $l=$ $58,91,100$ one finds $n_{\max }=1,2,3$, respectively in compliance with Eqs. (18) and (19). In fact if one considers the tables as the wave number plane $(l, n)$ the dashed portion of the plane is the forbidden zone for a normal mode to exist. Analogs of these features exist for the propagation of electromagnetic waves in optical fibers.

The frequencies, $\omega_{n m l}$ and the damping rates, $\alpha_{n m l}$, are given in Tables 4-6. Both are in units of the interior Alfvèn frequency. For a given $m$ the frequencies increase with increasing $n$ and/or $l$. The behavior with increasing $m$ is not so clear. For example, one observes that $\omega_{n, 0,100}>\omega_{n, 2,100}>\omega_{n, 1,100}$. The damping rates are smaller than the frequencies by a factor of about $S=R=2 \times 10^{4}$, a numerical attestation to the validity of the assumption of Eq. (21). They also increase with increasing $n$ and $l$.

\section{Graphic presentations}

To acquaint oneself with the characteristics of the magnetic and velocity fields of the modes we give some sample plots. By Eqs. (A3-4) $\delta v_{z}=0$, and $\delta v_{r}$ and $\delta v_{\phi}$ are proportional and 


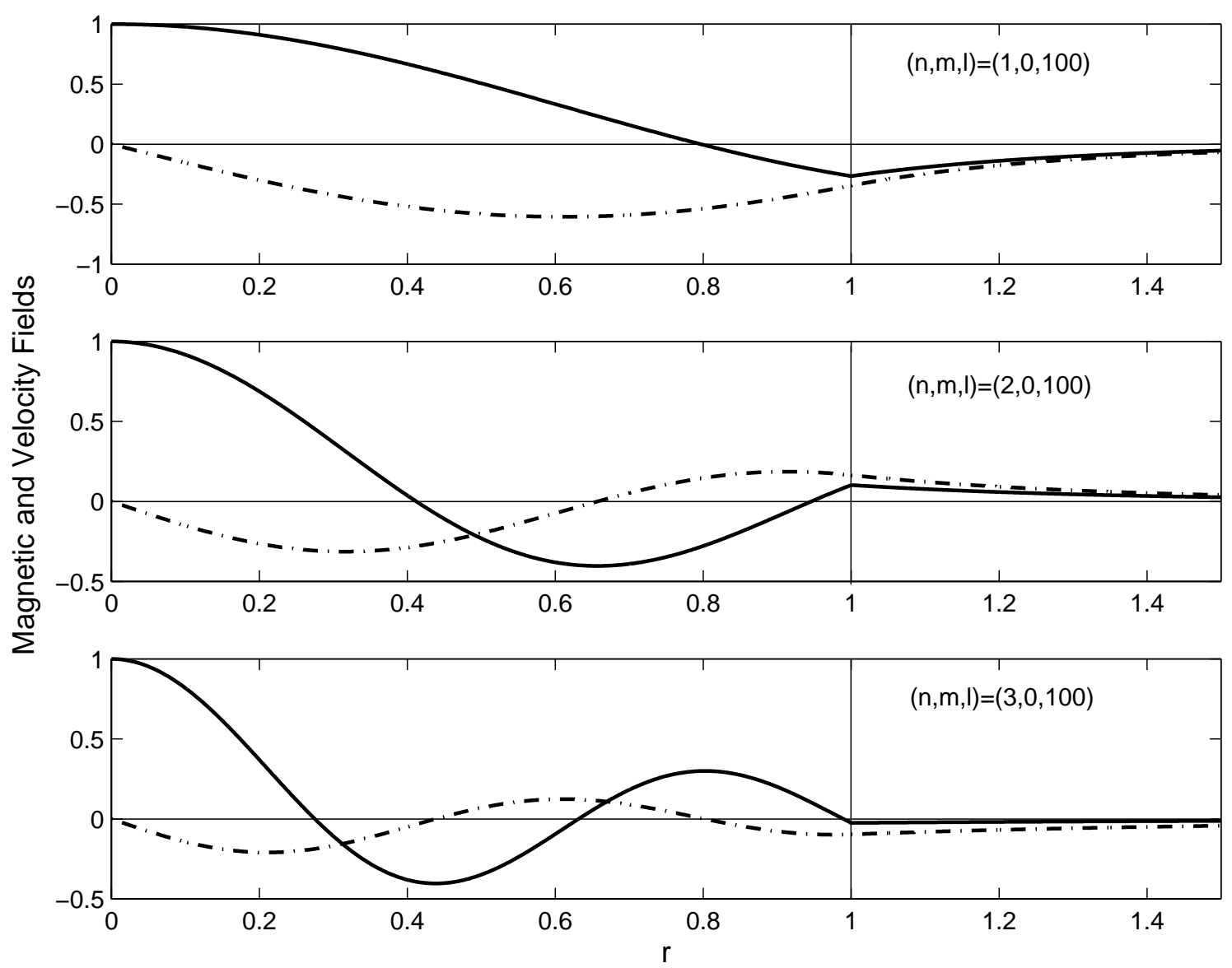

Fig. 2. Magnetic and velocity field components for $m=0$ (sausage) modes. $\delta B_{r}$ and $\left(-\delta v_{r}\right)$ dash-dotted curves; $\delta B_{z}$ solid curves. No $\delta B_{\phi}, \delta v_{\phi}$ and $\delta v_{z}$ for $m=0$. Auxiliary parameters are: $l=100, n=1,2,3 ; C_{100}^{2}=8.9$, radius $=10^{3} \mathrm{~km}$, length $=10^{5} \mathrm{~km}$, and $\rho_{\mathrm{e}} / \rho_{\mathrm{i}}=0.1$.

Table 4. Values of $\omega_{n m l}$ and $\alpha_{n m l}$ for $m=0$ (sausage) modes with radius $=10^{3} \mathrm{~km}$, length $=10^{5} \mathrm{~km}, B=100 \mathrm{G}, \rho_{\mathrm{i}}=2 \times 10^{-14} \mathrm{gr} \mathrm{cm}^{-3}$, $\rho_{\mathrm{e}} / \rho_{\mathrm{i}}=0.1, \omega_{\mathrm{A}}=2 \mathrm{rad} \mathrm{s}^{-1}$ and $S=R=2 \times 10^{4}$. Both frequencies, $\omega_{n m l}$ and the damping rates, $\alpha_{n m l}$, are in units of the interior Alfvèn frequency.

\begin{tabular}{ccccccc}
\hline \hline$l$ & & $\omega_{n m l}$ & & \multicolumn{3}{c}{$\alpha_{n m l}\left(\times 10^{-4}\right)$} \\
\hline 25 & - & - & - & - & - & - \\
26 & 2.5533 & - & - & 0.5188 & - & - \\
58 & 3.3171 & - & - & 0.8756 & - & - \\
59 & 3.3407 & 5.8337 & - & 0.8881 & 2.7081 & - \\
91 & 4.1264 & 6.4558 & - & 1.3550 & 3.3166 & - \\
92 & 4.1518 & 6.4746 & 9.1285 & 1.3717 & 3.3359 & 6.6311 \\
100 & 4.3571 & 6.6267 & 9.2916 & 1.5107 & 3.4947 & 6.8703 \\
\hline & $n=1$ & $n=2$ & $n=3$ & $n=1$ & $n=2$ & $n=3$ \\
\hline
\end{tabular}

opposite to $\delta B_{r}$ and $\delta B_{\phi}$, respectively. All components of $\delta \boldsymbol{B}$ and $\delta \boldsymbol{v}$ are plotted in Figs. 2 to 4 . In accordance with the boundary conditions of Eqs. (10), (11), the $r$ - and $z$-components are continuous at $r=1$ but have discontinuous slopes. The $\phi$ component and its slope are both discontinuous. The amplitudes are highly evanescent outside the tube and maybe neglected for all practical purposes, for instance, in considering the heating of corona. The number of nodes in each case is $n$.
Table 5. Same as Table 4, for $m=1$ (kink) modes.

\begin{tabular}{ccccccc}
\hline \hline$l$ & \multicolumn{3}{c}{$\omega_{n m l}$} & \multicolumn{3}{c}{$\alpha_{n m l}\left(\times 10^{-4}\right)$} \\
\hline 1 & 0.0434 & - & - & 0.0001 & - & - \\
40 & 1.4829 & - & - & 0.1750 & - & - \\
41 & 1.5154 & 4.0473 & - & 0.1827 & 1.3035 & - \\
74 & 2.5646 & 4.7377 & - & 0.5234 & 1.7862 & - \\
75 & 2.5957 & 4.7589 & 7.4084 & 0.5361 & 1.8022 & 4.3675 \\
100 & 3.3695 & 5.3079 & 7.8652 & 0.9035 & 2.2420 & 4.9228 \\
\hline & $n=1$ & $n=2$ & $n=3$ & $n=1$ & $n=2$ & $n=3$ \\
\hline
\end{tabular}

The antinodes of $\delta B_{z}$ coincide with the nodes of $\delta B_{r}$; for, $\delta B_{r}$ is proportional to the derivative of $\delta B_{z}$; see Eq. (A.1). The converse is, however, not true. The nodes of $\delta B_{\phi}$ and $\delta B_{z}$ occur at the same place, for they are proportional to each other. See Eqs. (17) and (A.2). For $m=1$, however, there is an exception. At $r=0, \delta B_{\phi}$ is finite and $\delta B_{z}$ has a node.

The velocity field has no $z$-component. Its transverse components, $\delta \boldsymbol{v}_{\perp}$, are proportional and opposite in direction to $\delta \boldsymbol{B}_{\perp}$, and have the same graphical representations. It should be remarked, however, that, because of $i$ in Eqs. (4), (6), (A.1) and (A.3) the phase of $\delta B_{r}$ and $\delta v_{r}$ differ from those of $\delta B_{z}$, $\delta B_{\phi}$, and $\delta v_{\phi}$ by $\pi / 2$. 

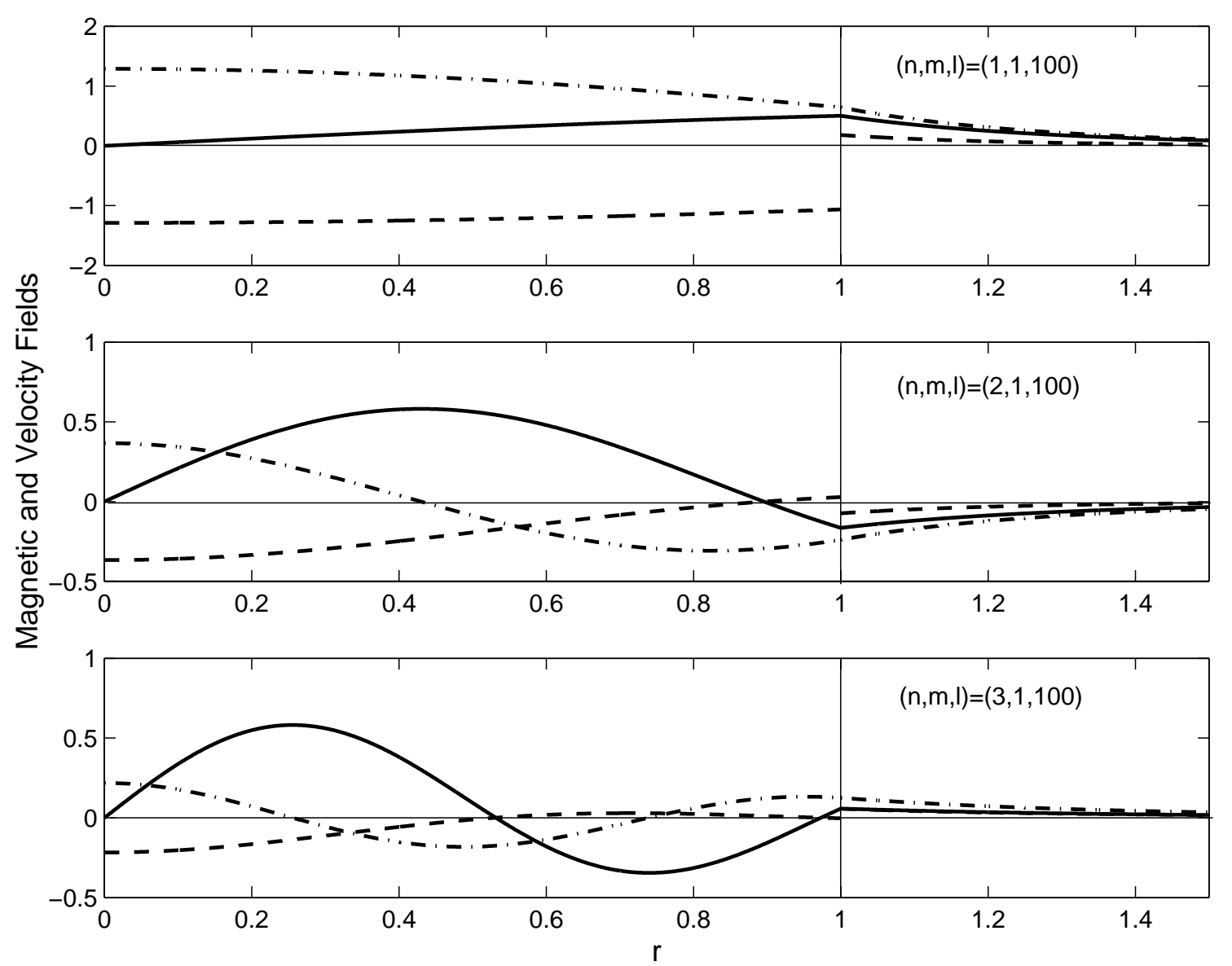

Fig. 3. Magnetic and velocity field components for $m=1$ (kink) modes. $\delta B_{r}$ and ( $\left.-\delta v_{r}\right)$ dash-dotted curves; $\delta B_{\phi}$ and $\left(-\delta v_{\phi}\right)$ dashed curves; $\delta B_{z}$ solid curves; no $\delta v_{z}$. Auxiliary parameters as in Fig. 2.

Table 6. Same as Table 4, for $m=2$ (fluting) modes.

\begin{tabular}{ccccccc}
\hline \hline$l$ & \multicolumn{3}{c}{$\omega_{n m l}$} & \multicolumn{4}{c}{$\alpha_{n m l}\left(\times 10^{-4}\right)$} \\
\hline 1 & 0.0434 & - & - & 0.0001 & - & - \\
54 & 2.1003 & - & - & 0.3510 & - & - \\
55 & 2.1350 & 5.4226 & - & 0.3627 & 2.3399 & - \\
89 & 3.2602 & 6.0567 & - & 0.8458 & 2.9192 & - \\
90 & 3.2922 & 6.0764 & 8.8849 & 0.8625 & 2.9382 & 6.2820 \\
100 & 3.6093 & 6.2766 & 9.0558 & 1.0366 & 3.1350 & 6.5260 \\
\hline & $n=1$ & $n=2$ & $n=3$ & $n=1$ & $n=2$ & $n=3$ \\
\hline
\end{tabular}

The densities of viscous and ohmic dissipation, Eq. (A.5) and the integrand in Eq. (24) are plotted in Fig. 5. Comparing Fig. 5 with Figs. 2 to 4 , one concludes that the highest and the lowest heating rates occur at the antinodes and nodes of $\delta B_{r}$ and $\delta v_{r}$, respectively. In regions exterior to the flux tube, $r>1$, the energy densities sharply drop to zero, supporting the assertion that the wave energy and heat dissipations are not of significance in the outer regions.

\section{Concluding remarks}

The modal structure of a magnetized flux tube under coronal conditions is investigated. The dispersion relation is solved in its most general form. The complete set of eigenfrequencies and eigenfields are calculated. Each mode is specified by a trio of wave numbers $(n, m, l)$, pertaining to the $(r, \phi, z)$ directions, respectively. Eigenfrequencies increase with increasing $n$ and $l$. For a given $m$, the wave number plane $(n, l)$ has a forbidden zone in which no eigenmode can exists. This sets an upper cutoff for $n$ and a lower cutoff for $l$. Asymptotic relations for determining these cutoffs are given. In the outer regions of the flux tube all components of $\delta \boldsymbol{B}$ and $\delta \boldsymbol{v}$ are much smaller and are highly evanescent. Therefore, one should not be surprised to find out that much of the heat is also generated within the tube.

For weak viscous and ohmic dissipations, time-decay exponents are calculated for each mode. The density of heat production rates as functions of $r$ are the same for both mechanisms. Their contributions, however, are inversely proportional to the Reynolds and Lundquist numbers, $R$ and $S$, respectively. The total dissipation time scale becomes proportional to $\left(R^{-1}+S^{-1}\right)$. The total generated heat is, of course, equal to the total initial energy of the wave. We cannot asses the actual values of resistivity and viscosity prevailing in coronal regions. One, however, finds the following values quoted in the literature:

Steinolfson \& Davila (1993) use the values $S=10^{3}, 10^{4}$, and $10^{5}$. Ofman et al. (1994) assume $S=10^{4}$ and $R=560$ in active coronal regions and $S=10^{4}$ and $R=0.56$, otherwise. Viscosity in their analysis is compressional. 


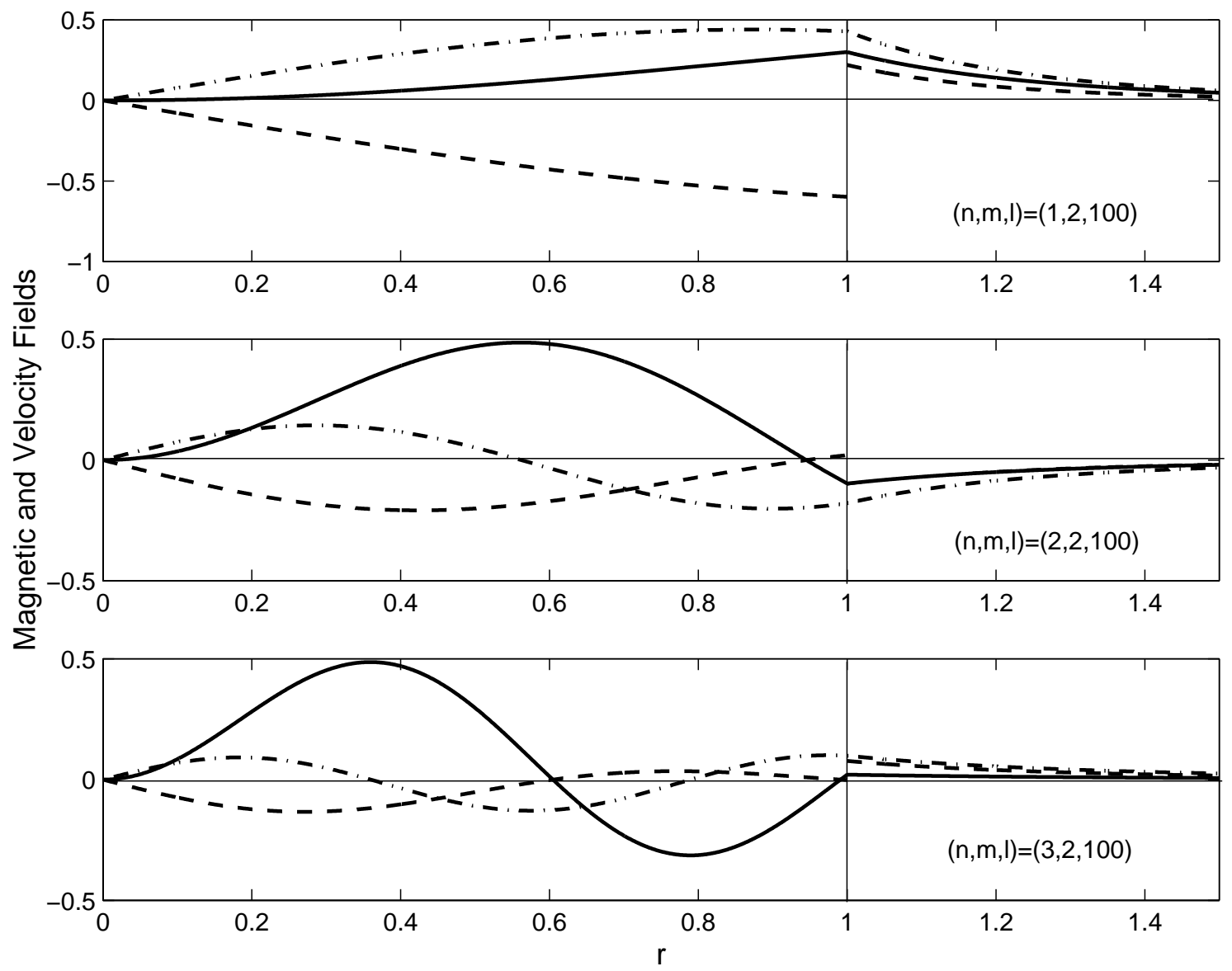

Fig. 4. Magnetic and velocity field components for $m=2$ (fluting) modes. Legend and auxiliary parameters as in Fig. 3.

Nakariakov et al. (1999) predict $S=10^{13}$ and $R=10^{14}$ on theoretical grounds and report $S=10^{5-5.8}$ and $R=10^{5.3-6.1}$ from observational evidence.

The values $S=R=2 \times 10^{10}$ employed in the present paper is for an academic exercise and no further significance should be attached to it.

Acknowledgements. This work was supported by the Institute for Advanced Studies in Basic Sciences, Zanjan. The authors wish to thank Prof. Wentzel, Prof. Ofman and Dr. Nakariakov for providing valuable consultations.

\section{Appendix A: Some useful formulas}

The key to the analysis of a mode is $\delta B_{z, n m l}$ of Eq. (17). Substituting this in Eq. (4) gives the remaining components of $\delta \boldsymbol{B}$. Thus,

$$
\begin{aligned}
\delta B_{r, n m l} & =\frac{i l}{L x_{n m l}^{2}} \frac{\mathrm{d} J_{m}\left(x_{n m l} r\right)}{\mathrm{d} r} \mathrm{e}^{i\left(l z / L+m \phi-\omega_{n m l} t\right)}, \quad r<1, \\
& =-\frac{i l}{L y_{n m l}^{2}} \frac{\mathrm{d} K_{m}\left(y_{n m l} r\right)}{\mathrm{d} r} A_{n m l} \mathrm{e}^{i\left(l z / L+m \phi-\omega_{n m l} t\right)}, \quad 1<r, \\
\delta B_{\phi, n m l} & =-\frac{l}{L x_{n m l}^{2}} \frac{m}{r} J_{m}\left(x_{n m l} r\right) \mathrm{e}^{i\left(\left(l z / L+m \phi-\omega_{n m l} t\right)\right.}, \quad r<1, \\
& =\frac{l}{L y_{n m l}^{2}} \frac{m}{r} A_{n m l} K_{m}\left(y_{n m l} r\right) \mathrm{e}^{i\left(l z / L+m \phi-\omega_{n m l} t\right)}, \quad 1<r .
\end{aligned}
$$

By Eqs. (4) and (6) $\delta \boldsymbol{v}_{\perp}$ is parallel to $\delta \boldsymbol{B}_{\perp}$, and by Eq. (5) $\delta v_{z}$ is zero. Thus,

$\delta \boldsymbol{v}_{\perp, n m l}=-\left(L \omega_{n m l} / l B\right) \delta \boldsymbol{B}_{\perp, n m l}$,

$\delta v_{z, n m l}=0, \quad 0<r<\infty$.

The ohmic and viscous dissipation densities of Eq. (24) is

$(S / 2 \sigma)\left|\delta \boldsymbol{j}_{n m l}(r)\right|^{2}=(R \eta / 2)\left|\delta \boldsymbol{v} \cdot \nabla^{2} \delta \boldsymbol{v}\right|$

$=\left(\omega_{\mathrm{A}} / 16 \pi^{2}\right)\left[\left(l / L x_{n m l}\right)^{2}+1\right]^{2} I_{\mathrm{i}}, \quad r<1$,

$=\left(\omega_{\mathrm{A}} / 16 \pi^{2}\right) A_{n m l}^{2}\left[\left(l / L y_{n m l}\right)^{2}-1\right]^{2} I_{\mathrm{e}}, \quad 1<r$.

The energy density of wave (dimensionless), the sum of kinetic and magnetic energy densities is

$$
\begin{aligned}
\varepsilon & =\frac{1}{2}\left\{\frac{1}{2} \rho\left|\delta \boldsymbol{v}_{n m l}\right|^{2}+\frac{1}{8 \pi}\left|\delta \boldsymbol{B}_{n m l}\right|^{2}\right\}, \\
& =\frac{1}{16 \pi}\left\{\left[2\left(l / L x_{n m l}\right)^{2}+1\right]\left(I_{\mathrm{i}} / x_{n m l}^{2}\right)+J_{m}^{2}\left(x_{n m l} r\right)\right\}, \quad r<1, \\
& =\frac{1}{16 \pi} A_{n m l}^{2}\left\{\left[2\left(l / L y_{n m l}\right)^{2}-1\right]\left(I_{\mathrm{e}} / y_{n m l}^{2}\right)+K_{m}^{2}\left(y_{n m l} r\right)\right\}, \quad 1<r .
\end{aligned}
$$

The expressions $I_{\mathrm{i}}$ and $I_{\mathrm{e}}$ in Eqs. (A.5), (A.6) are

$I_{\mathrm{i}}=\left(\frac{\mathrm{d} J_{m}\left(x_{n m l} r\right)}{\mathrm{d} r}\right)^{2}+\frac{m^{2}}{r^{2}} J_{m}^{2}\left(x_{n m l} r\right)$, 

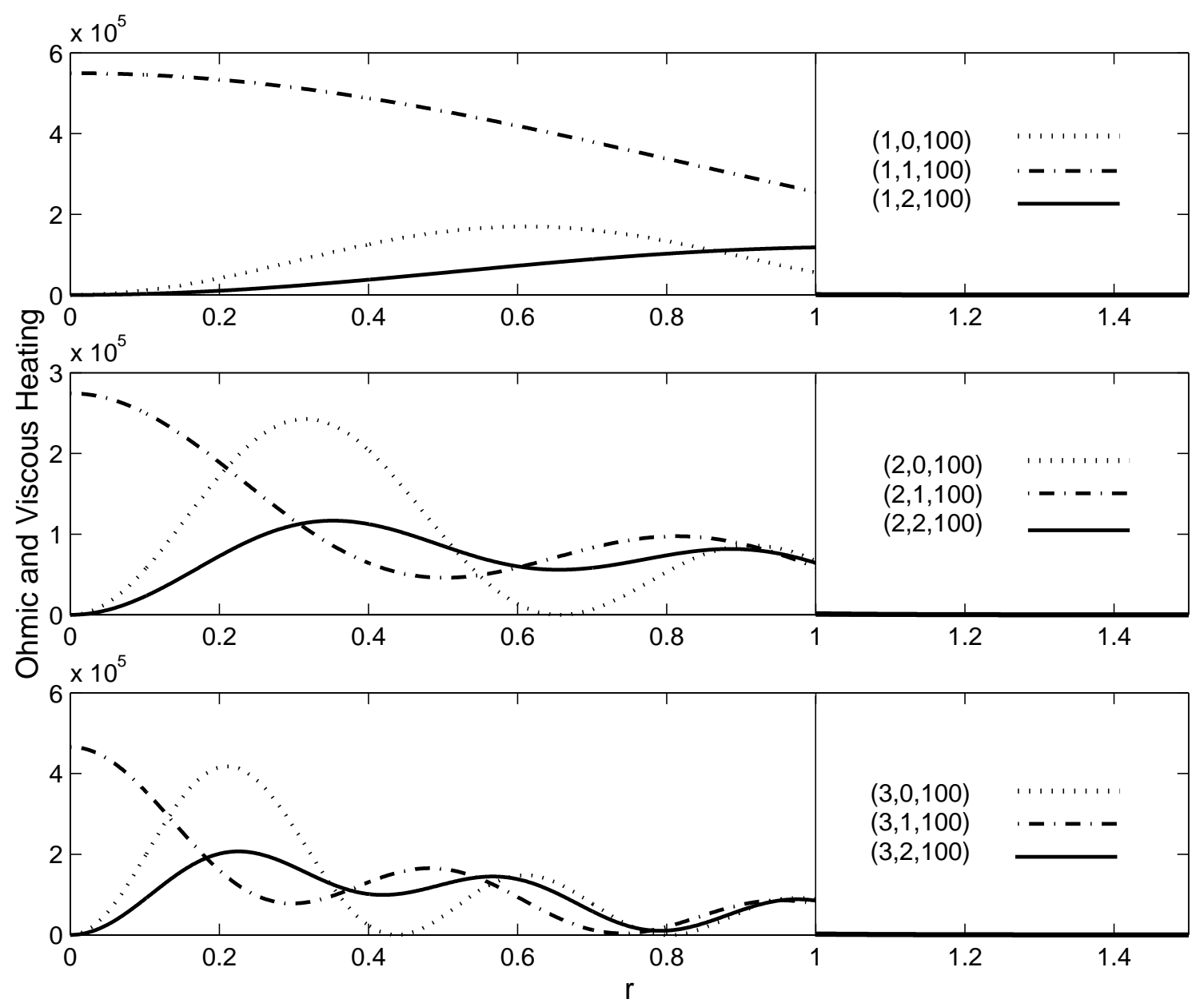

Fig. 5. Densities of ohmic and viscous dissipations $(S / 2 \sigma)\left|\delta \boldsymbol{j}_{n m l}(\boldsymbol{r})\right|^{2}=(R \eta / 2)\left|\delta \boldsymbol{v} \cdot \nabla^{2} \delta \boldsymbol{v}\right|$ in Eq. (A.5), values of $(n, m, l)$ and legend are given in the right hand caption. Auxiliary parameters are $C_{100}^{2}=8.9$, radius $=10^{3} \mathrm{~km}$, length $=10^{5} \mathrm{~km}, \rho_{\mathrm{e}} / \rho_{\mathrm{i}}=0.1, \omega_{\mathrm{A}}=2 \mathrm{rad} \mathrm{s} \mathrm{s}^{-1}$, and $S=R=2 \times 10^{4}$. In $r>1$ dissipation is much small and does not show up in the plots.

$I_{\mathrm{e}}=\left(\frac{\mathrm{d} K_{m}\left(y_{n m l} r\right)}{\mathrm{d} r}\right)^{2}+\frac{m^{2}}{r^{2}} K_{m}^{2}\left(y_{n m l} r\right)$

In calculating the total heat or energy of a mode, the third equality in Eq. (24), the following integrals are needed.

$\int_{0}^{1} I_{\mathrm{i}} r \mathrm{~d} r=x J_{m}(x) J_{m}^{\prime}(x)+\frac{x^{2}}{2}\left\{J_{m}^{\prime 2}(x)+\left(1-\frac{m^{2}}{x^{2}}\right) J_{m}^{2}(x)\right\}$,

$\int_{1}^{\infty} I_{\mathrm{e}} r \mathrm{~d} r=-y K_{m}(y) K_{m}^{\prime}(y)-\frac{y^{2}}{2}\left\{K_{m}^{\prime 2}(y)-\left(1+\frac{m^{2}}{y^{2}}\right) K_{m}^{2}(y)\right\}$

$$
y=y_{n m l} \text {. }
$$

As noted earlier, substituting Eqs. (A.5) and (A.6) in Eq. (24), after some integrations by parts reveals that the total dissipation over all times exactly equals the total initial energy of the wave.

\section{Appendix B: Transverse Alfvèn modes}

Setting $\delta B_{z}=0$ in Eqs. (1)-(2) in the absence of dissipation leads to

$\frac{\partial^{2} \delta \boldsymbol{B}_{\perp}}{\partial t^{2}}=v_{\mathrm{A}}^{2} \frac{\partial^{2} \delta \boldsymbol{B}_{\perp}}{\partial z^{2}}$
Similar equation holds for $\delta \boldsymbol{v}_{\perp}$. Hollweg (1984), Roberts \& Ulmschneider (1997), Kivelson \& Russell (1997), and Nakariakov (2000) have passing remarks on these transverse, or what is commonly called torsional, waves. Here we wish to add a few details. The solution of Eq. (B.1) corresponding to rigid boundary conditions at $z=0$ and $\pi L$ is

$\delta \boldsymbol{B}_{\perp}=\delta \boldsymbol{B}_{\mathrm{i}, \mathrm{e}}\left(\boldsymbol{r}_{\perp}\right) \mathrm{e}^{i\left(l z / L-\omega_{\mathrm{i}, \mathrm{e}} t\right)}$,

where $i$ and $e$ refer to the interior and exterior of the flux tube. In particular $\omega_{\mathrm{i}}^{2}=v_{\mathrm{A}_{\mathrm{i}}}^{2} l^{2} / L^{2}$ and $\omega_{\mathrm{e}}^{2}=v_{\mathrm{A}_{\mathrm{e}}}^{2} l^{2} / L^{2}$. Let $c\left(\boldsymbol{r}_{\perp}\right)=0$ be the boundary of the flux tube in the plane transverse to the $z$-axis. Continuity of the normal component of $\delta \boldsymbol{B}$ requires

$\boldsymbol{n} \cdot\left[\delta \boldsymbol{B}_{\mathrm{i}}(c) \mathrm{e}^{-i \omega_{\mathrm{i}} t}-\delta \boldsymbol{B}_{\mathrm{e}}(c) \mathrm{e}^{-i \omega_{\mathrm{e}} t}\right]=0$,

where $\boldsymbol{n}$ is a unit vector normal to $c$. The only way to satisfy Eq. (B.3) for all times is to let

$\delta \boldsymbol{B}_{\mathrm{i}}(c)=\delta \boldsymbol{B}_{\mathrm{e}}(c)=0$.

That is to say, disturbances inside and outside the tube are not coupled and evolve independently. In fact there are no dynamics to determine the dependence of the wave on $\boldsymbol{r}_{\perp}$. Any arbitrary transverse disturbance in magnetic and velocity fields can 
propagate in the $z$-direction with the Alfvèn speed. In the absence of resistivity and viscosity, propagation is dispersionless and dissipationless. Resistivity and viscosity causes the wave to disperse and dissipate away.

\section{References}

Davila, J. M. 1987, ApJ, 317, 514

Edwin, P. M., \& Roberts, B. 1983, Sol. Phys., 88, 179

Ghatak, A., \& Thyagarajan, K. 1998, Inroduction to Fiber Optics (Cambridge Univ. Press), 132

Hasan, S. S., \& Sobouti, Y. 1987, MNRAS, 228, 427

Hollweg, J. V. 1984, ApJ, 277, 392

Ionson, J. A. 1978, ApJ, 226, 650

Kivelson, M. G., \& Russel, C. T. 1997, Introduction to Space Physics (Cambridge Univ. Press)

Nakariakov, V. M., \& Roberts, B. 1995, Sol. Phys., 159, 399

Nakariakov, V. M., Ofman, L., Deluca, E. E., Roberts, B., \& Davila, J. M. 1999, Science, 285, 862
Nakariakov, V. M. 2000, Magnetohydrodynamic Waves in Solar Coronal Structures, http://www.astro.warwick.ac.uk/ $\sim$ valery/mhdwaves

Nasiri, S. 1992, A\&A, 261, 615

Ofman, L., Davila, J. M., \& Steinolfson, R. S. 1994, ApJ, 421, 360

Ofman, L., Davila, J. M., \& Steinolfson, R. S. 1995, ApJ, 444, 471

Roberts, B. 1981a, Sol. Phys., 69, 27

Roberts, B. 1981b, Sol. Phys., 69, 39

Roberts, B., Edwin, P. M., \& Benz, A. O. 1984, ApJ, 279, 857

Roberts, B., \& Ulmschneider, P. 1997, in Lecture Notes in Physics, ed. G. M. Simnett, C. E. Alissandrakis, \& L. Vlahos (Springer), 75 Steinolfson, R. S., \& Davila, J. M. 1993, ApJ, 415, 354

Steinolfson, R. S., Priest, E. R., Poedts, S., Nocera, L., \& Goossens, M. 1986, ApJ, 304, 526

Wentzel, D. G. 1979a, ApJ, 227, 319

Wentzel, D. G. 1979b, A\&A, 76, 20

Wilson, P. R. 1979, A\&A, 71, 9

Wilson, P. R. 1980, A\&A, 87, 121 